\title{
Estratificação de risco para predisposição de doenças renais crônicas em um grupo de idosos
}

\author{
Risk stratification for predisposition of chronic renaissance diseases \\ in an elderly group
}

\author{
Analdo Costa Alfaia Júnior' • Ivaldo José Gentil Pantoja² • Eliana Caldas Lisboa ${ }^{3}$ \\ Camilo Eduardo Almeida Pereira ${ }^{4} \bullet$ Marcela Raissa Asevedo Dergan ${ }^{5}$ Lidiane Assunção de Vasconcelos $^{6}$
}

\begin{abstract}
RESUMO
Objetivo: analisar o estilo de vida da população idosa que predispõe as doenças renais crônicas. Método: Esse estudo é descritivo com abordagem quantitativa, realizado com 286 idosos de uma unidade básica de saúde do município de Belém. Resultado :O p-valor é significativo para a correlação de antecedentes $x$ conhecimento. Em relação o escore de predisposição para doença renal a prevalência foi de 40-49,9 pontos, classificando os participantes em grau $\vee$ para o risco de predisposição de doença renais crônicas. Conclusão:A realização da classificação de risco para doenças renais crônica é uma ferramenta importante para estabelecer um plano terapêutico singular, condizente com a necessidade de cada usuário.
\end{abstract}

Palavras-chave: Idoso; Fatores de Riscos; Doença Renal Crônica.

\begin{abstract}
Objective:To analyze the lifestyle of the elderly population that predisposes to chronic kidney disease. Method:This is a descriptive study with a quantitative approach, conducted with 286 elderly from a basic health unit in the city of Belém. Result:The $\mathrm{p}$-value is significant for the correlation of antecedents $\mathrm{x}$ knowledge. Regarding the predisposition score for kidney disease the prevalence was 40-49.9 points, classifying the participants in grade $V$ for the risk of predisposition to chronic kidney disease. Conclusion: The achievement of chronic kidney disease risk classification is an important tool to establish a unique therapeutic plan, consistent with the needs of each user.
\end{abstract}

Keywords: Elderly; Risk factors; Chronic Kidney Disease.

I Graduado em Enfermagem pela Faculdade Pan Amazônica

2 Graduado em Enfermagem pela Faculdade Pan Amazônica

3 Graduado em Enfermagem pela Faculdade Pan Amazônica

4 Graduação em enfermagem pela Universidade do Estado do Pará (2014) e mestrado em Saúde, Ambiente e Sociedade na Amazônia pela Universidade Federal do Pará (2017) e Especialização em Enfermagem do Trabalho pela Faculdade Integrada do Rio de Janeiro.Atualmente é professor adjunto da Faculdade Pan Amazônica e Faculdade Paraense de Ensino, Professor colaborador da Faculdade de Medicina da Universidade Federal do Pará, Preceptor do curso de medicina da Universidade do Estado do Pará; Bolsista no curso de Especialização Saúde da Família- UFPA.

5 Graduado em Enfermagem pela Faculdade Pan Amazônica

6 Possui graduação em Enfermagem pela 6Universidade Federal do Pará (2009) e Mestrado em Saúde,Ambiente e Sociedade na Amazónia pela Universidade Federal do Pará (2016).Atualmente é professora substituta da Universidade Federal do Pará, lotada na Faculdade de Medicina do Pará- UFPA e da Universidade do Estado Pará na Faculdade de Enfermagem, Coordenadora dos Cursos da Saúde e tutora EAD no Curso especialização Saúde da Família- UFPA e Preceptora de Ensino no Eixo/ Unidade Curricular : Interação Ensino, Serviço, Comunidade e Gestão. 


\section{INTRODUÇÃO}

As Doenças Renais Crônicas (DRC) estão cada vez mais prevalentes nos países desenvolvidos e em desenvolvimentos, segundo o Instituto Brasileiro de Nefrologia, e com grandes chances de serem adquiridas na terceira idade, com complicações de diversos estados patológicos, que alteram o equilíbrio dos eletrólitos ou ácido-base, tornando os rins incapazes de proceder à eliminação de certos resíduos produzidos no organismo (homeostase), e consequentemente a perda de suas funções ${ }^{(1)}$.

Autores destacam que cerca de $63 \%$ dos casos de DRCs, a Hipertensão e a Diabetes Mellitus tipo 2 estão presentes. Estudos relacionados ao tema têm mostrado que a hipertensão arterial sistêmica (HAS) e a função renal estão intimamente relacionadas, podendo a hipertensão não apenas ser a causa como também a consequência da disfunção renal(2-3).

Segundo o Ministério da Saúde, o número de pacientes portadores de doenças renais está aumentando em todo o mundo. Nos Estados Unidos da América, estima-se um crescimento anual de $6 \%$ de novos casos e que dobra a cada 10 anos. No Brasil, embora os números sejam mais modestos e nem sempre precisos, não deixam de ser preocupantes, principalmente no meio da população idosa, visto que o país passa por uma transição demográfica em decorrência da diminuição da taxa de natalidade e o aumento da expectativa de vida, porém essa transição não necessariamente é acompanhada de um envelhecimento saudável ${ }^{(4-5)}$.

O processo de envelhecimento construído através da longevidade saudável está relacionado ao estilo de vida que essa população se submete, pois, por mais, que sejam portadores de doenças crônicas não transmissíveis, como as DRCs, podem ter uma expectativa de vida estável, em sua geração, pois o aumento da população idosa é uma realidade que exige mudança dos paradigmas e estratégias políticas, econômicas e sociais, potencializados por fatores condicionantes e ambientais ${ }^{(6)}$.

Assim é necessário compreender a relação do estilo de vida da população idosa com as DRCs, já que estatísticas indicam que os índices de DRCs têm aumentado em meio à população idosa, configurando-se como um problema de saúde pública, uma vez que são agravos que acarretam a qualidade de vida da população, além de representar um alto custo para o Sistema Único de Saúde. Desta forma, esse estudo temo como objetivo analisar o estilo de vida da população idosa que predispõe as doenças renais crônicas.

\section{METODOLOGIA}

Este estudo é de caráter descritivo exploratório, com abordagem quantitativa. Foi realizada na Unidade Básica de Saúde (UBS), pertencente ao Distrito Administrativo Guamá (DAGUA), vinculada à Secretaria Municipal de Saúde (SESMA), localizada na cidade de Belém-Pará, com atendimento restrito à população cadastrada, pertencente ao território.

Constatou-se que I.822 eram acompanhados pelas equipes I, 2 e 3, compostas na UBS, e, por conseguinte 852 entraram nos critérios de exclusão, por não terem capacidade cognitiva preservada ou apresentarem alguma limitação locomotiva. Para o cálculo do tamanho da amostra foi prefixado o erro amostral em $5 \%$, conforme a aplicação da técnica amostral de Cochran (7), ao final, o número da amostra foi de 286 idosos. Por se tratar de uma pesquisa que envolve seres humanos, o mesmo foi analisado pelo Comitê de Ética da Universidade Paulista, para verificar se a pesquisa não infringiria os princípios éticos e legais dos sujeitos pesquisados, após análise do comitê o projeto foi aprovado sob o parecer 2.962.423.

Para avaliar os dados foram aplicados métodos estatísticos descritivos e inferenciais. As avaliações das variáveis qualitativas foram apresentadas por distribuições de frequências absolutas e relativas. As variáveis quantitativas foram apresentadas por medidas de tendência central e de variação. A comparação da frequência dos eventos que determinam a pré-disposição para DRC foi realizada pelo teste Binomial.

No que tange avaliação da correspondência entre os componentes do PRÉ-DRC (Conhecimento, Antecedentes e Estilo de vida) foi aplicada a correlação linear de Pearson. A avaliação do escore PRÉ-DRC conforme o Sexo foi realizada pelo teste $t$ de Student e avaliação do escore PRÉ-DRC conforme Estado civil, e Renda familiar foi realizada pela ANOVA (Análise de Variância).

A correlação entre o PRÉ-DRC e a Idade e IMC foi realizada pela correlação linear de Pearson. Foi previamente fixado o nível de significância alfa $=0.05$ (margem de erro alfa de 5\%) para rejeição da hipótese de nulidade. $O$ planejamento da análise de dados foi realizado no software dEASYgner versão 3.0. O processamento estatístico foi realizado no software BioEstat versão 5.3.

\section{RESULTADO}

O presente estudo avaliou os dados de 286 idosos, sendo $59.1 \%$ do sexo Feminino e $40.9 \%$ do sexo Masculino. A cor da pele predominante é parda $87.8 \%$ e o estado civil Casado (55.6\%) é o mais frequente. A idade dos pacientes variou entre 60 e 86 anos, sendo a média 65.5 anos com desvio padrão de 5.5 anos. Esses idosos são $55 \%$ aposentados, tem ocupação 
TABELA 1 - Características gerais dos idosos da UBS Condor. Belém/PA, ano 2018.

\begin{tabular}{lcc}
\hline Categoria & N & $\%$ \\
\hline Sexo & 117 & 40.9 \\
Masculino & 169 & 59.1 \\
Feminino & & 4.2 \\
Cor da pele & 12 & 87.8 \\
Branca & 251 & 7.7 \\
Parda & 22 & 0.3 \\
Negra & 1 & 0.0 \\
Amarela & 0 & 9.1 \\
Indígena & & 9.1 \\
Estado Civil & 26 & 55.6 \\
Solteiro & 159 & 14.3 \\
Casado & 41 & 1.0 \\
Viúvo & 3 & 19.9 \\
Divorciado & 57 & \\
U Estável & & \\
\hline
\end{tabular}

Fonte: Elaborado pelos autores, Belém, 2018.

TABELA 2 - Idade e IMC dos 286 idosos da UBS Condor. Belém/PA, ano 2018.

\begin{tabular}{lcc}
\hline & Idade (anos) & IMC (kg/cm2) \\
\hline Tamanho da amostra & 286 & 286 \\
Mínimo & 60.0 & 18.4 \\
Máximo & 86.0 & 47.5 \\
Mediana & 64.0 & 26.4 \\
Primeiro Quartil & 61.0 & 24.2 \\
Terceiro Quartil & 68.0 & 28.9 \\
Média Aritmética & 65.5 & 27.0 \\
Desvio Padrão & 5.5 & 4.5 \\
\hline
\end{tabular}

I8\% do Lar (sexo feminino) e $27 \%$ tem outras ocupações. A renda familiar predominante é dois salários mínimos (52\%), os que ganham um salário mínimo são $37 \%$ e apenas II\% ganham mais que dois salários mínimos. Essas características gerais podem ser verificadas na tabela $\mathrm{I}$.

Tabela 2 mostra a relação entre Idade e IMC. No que se refere ao IMC dos participantes, o valor mínimo encontrado foi $18.4 \mathrm{~kg} / \mathrm{cm} 2$, a máxima foi $47.5 \mathrm{~kg} /$ $\mathrm{cm} 2$ e a mediana $26.4 \mathrm{~kg} / \mathrm{cm} 2$, tendo como media aritmética $27.0 \mathrm{~kg} / \mathrm{cm} 2 \mathrm{com}$ desvio padrão de $4.5 \mathrm{~kg} / \mathrm{cm} 2$. Em relação à idade dos participantes, a mínima foi 60 anos e a máxima 86 anos e a mediana 64 anos, sendo a média aritmética 65.5 anos com desvio padrão de 5.5 anos.

Em relação aos antecedentes, 52,1\% dos entrevistados relataram ter algum membro da família com HAS. $57,0 \%$ são hipertensos e $44,0 \%$ fazem controle da HAS. Quando perguntados sobre seus conhecimentos ape- nas $15,5 \%$ declaram saber as causa que podem levar a doenças renais, apenas $10,9 \%$ referiram ter recebido alguma informação sobre doenças renais por profissionais da área de saúde e 10,2\% disseram saber como prevenir a doença renal.

No que se refere aos fatores de risco que podem levar a DRC, somente I,8\% citaram a automedicação, I,8\% disseram que o cigarro pode ser um fator de risco e 6,7\% mencionaram a bebida alcoólica. Quanto ao estilo de vida dos idosos, $66,2 \%$ relataram comer produtos industrializados, $96,8 \%$ consomem carne vermelha com uma frequência de três vezes ou mais na semana e 5I,4\% disseram não praticar atividade física.

Essas informações encontram-se na tabela 3, que trata sobre os determinantes e condicionantes que tem uma correlação com as DRC, ressalta que os dados foram agrupados por antecedentes, conhecimento e estilo de vida.

Analise do escore demonstra que a média para o 
TABELA 3 - Ocorrência de itens que determinam a pré-disposição para DRC em 286 idosos da UBS Condor. Belém/PA, ano 2018.

\begin{tabular}{|c|c|c|c|}
\hline Componentes do PRÉ-DRC & $\mathbf{N}$ & $\%$ & p-valor \\
\hline \multicolumn{4}{|l|}{ ANTECEDENTES } \\
\hline PII 01 - Doença Renal & 17 & 6.0 & \\
\hline PII 02 - Familiar de Renal & 21 & 7.4 & \\
\hline PII 03 - Familiar HAS & 148 & 52.1 & $<0.0001^{*}$ \\
\hline PII 04 - Tem HAS & 162 & 57.0 & $<0.0001^{*}$ \\
\hline PII 05 - Controla HAS & 125 & 44.0 & $<0.0001^{*}$ \\
\hline PII 06 - Familiar DM & 41 & 14.4 & \\
\hline PII 07 - Tem DM & 42 & 14.8 & \\
\hline PII 08 - Controla DM & 42 & 14.8 & \\
\hline PII 09 - Problema cardiovascular & 22 & 7.7 & \\
\hline \multicolumn{4}{|l|}{ CONHECIMENTO } \\
\hline PIII 01 - Declara saber as causas & 44 & 15.5 & $<0.0001^{*}$ \\
\hline PIII 02 - Recebeu informação & 31 & 10.9 & $<0.0001^{*}$ \\
\hline PIII 03 - Sabe como prevenir & 29 & 10.2 & $<0.0001^{*}$ \\
\hline PIII 04 - Citou 3 fatores de risco & 264 & 93.0 & \\
\hline Causa - DM e HAS & 143 & 50.4 & \\
\hline Causa - Alimentação & 264 & 93.0 & \\
\hline Causa - Falta de atividade física & 52 & 18.3 & \\
\hline Causa - Automedicação & 5 & 1.8 & $<0.0001^{*}$ \\
\hline Causa - Obesidade & 73 & 25.7 & \\
\hline Causa - Baixo consumo água & 272 & 95.8 & \\
\hline Causa - Cigarro & 5 & 1.8 & $<0.0001^{*}$ \\
\hline Causa - Bebida alcoólica & 19 & 6.7 & $<0.0001^{*}$ \\
\hline \multicolumn{4}{|l|}{ ESTILO DE VIDA } \\
\hline PIV 1 - Bebida alcoólica & 81 & 28.5 & \\
\hline PIV 2 - Fumante ou ex-fumante & 122 & 43.0 & $0.0002^{*}$ \\
\hline PIV 3 - Come Produto industrializado & 188 & 66.2 & $<0.0001^{*}$ \\
\hline PIV 4 - Come Comida salgada & 80 & 28.2 & \\
\hline PIV 5 - Come Carne vermelha & 275 & 96.8 & $<0.0001^{*}$ \\
\hline PIV 6 - Não pratica atividade física & 146 & 51.4 & $<0.0001^{*}$ \\
\hline PIV 7 - Água, pouco consumo & 33 & 11.6 & \\
\hline PIV 8 - Automedicação & 95 & 33.5 & \\
\hline
\end{tabular}

Fonte: Elaborado pelos autores, Belém, 2018.

* Comparações pelo teste Binomial

TABELA 4 - Componentes do PRÉ-DRC dos 286 idosos da UBS Condor. Belém/PA, ano 2018.

\begin{tabular}{lcccc}
\hline & Antecedentes & Conhecimento & Estilo de Vida & PRÉ-DRC \\
\hline Mínimo & 22.2 & 0.0 & 11.1 & 28.7 \\
Máximo & 77.8 & 58.3 & 100.0 & 70.4 \\
Amplitude Total & 55.6 & 58.3 & 88.9 & 41.7 \\
Mediana & 33.3 & 33.3 & 66.7 & 44.4 \\
Primeiro Quartil & 22.2 & 33.3 & 44.4 & 40.7 \\
Terceiro Quartil & 33.3 & 33.3 & 77.8 & 51.9 \\
Média Aritmética & 31.9 & 34.9 & 60.4 & 45.5 \\
Desvio Padrão & 10.7 & 8.5 & 19.1 & 7.5 \\
\hline
\end{tabular}

Fonte: Elaborado pelos autores, Belém, 2018.

Fórmula:

PRÉDRC $=\{[$ Antecedentes $+(100-$ Conhecimento $)+$ Estilo de Vida $] / 300\} \times 100$

Antecedentes: Zero é o melhor e 100 é pior.

Conhecimento: 100 é o melhor e 0 é pior.

Estilo de vida: Zero é o melhor e 100 é pior. 
antecedente é de 31.9 , evidenciando que os participantes tem uma percepção que esse aspecto é um fator de risco para as DRC, já em relação ao conhecimento dos participantes obtiveram uma pontuação baixa, ficando com a média de 34.9 , ou seja, os idosos não apresentam o nível satisfatório de conhecimento da doença, prevenção e os fatores de riscos. No que ser refere ao estilo de vida dos participantes, a média do escore de pontuação foi de 60.4 , o escore que se aproxima da pontuação máxima de 100 , que representa um estilo de vida que predispõe as DRC, conforme a tabela 4.

Ressalta que a pontuação é de um ponto para cada pergunta, de forma que para $\circ$ antecedente a pontuação máxima é de 9 , uma vez que utilizou-se as seguinte questões: antecedente familiar (doença renal, HAS, DM), antecedentes pessoais (doença renal, HAS, DM, problema cardíaco) e controle da HAS e DM, no quesito "controle" o usuário só pontuará se não fizer o controle, essas questões foram aplicadas com base na relevância desses fatores associados as DRC.

Para o conhecimento quanto maior a pontuação do usuário menor o risco de DRC, no entanto para receber a pontuação o participante tinha que saber as causas, ter recebido informações, saber a prevenção, saber os fatores de riscos (DM e HAS, Alimentação, Falta de atividade física, Automedicação, Obesidade, Baixo consumo de água, Cigarro, Bebida alcoólica), caso o usuário tenha citado três ou mais fatores de risco, o mesmo recebeu uma pontuação extra, desta forma a pontuação máxima para esse escore é de 12.

Já em relação ao estilo de vida, o participante só pontuou se tinha algum habito de risco para DRC (Bebida alcoólica, Fumante ou ex-fumante, come produto industrializado, Gosta de comida salgada, Come carne vermeIha, Não pratica atividade física, Água, pouco consumo, Automedicação). Referente ao consumo de carne vermelha, o participante só pontuou aqueles que relataram o consumo de três vezes ou mais na semana, sendo que a pontuação máxima é de 8 .
Para chegar no score é preciso fazer os seguintes cálculos:

Cálculo de cada Fator:

ANTECEDENTES: (Soma/9) $\times 100$

CONHECIMENTO: (Soma//2) $\times 100$

ESTILO DEVIDA: (Soma/8)xI00

Fórmula da predisposição:

PRÉDRC $=\{[$ Antecedentes $+(100-$ Conhecimento $)$ + Estilo de Vida] $/ 300\} \times 100$

Aplicando a correlação linear de Pearson, a fim de fazer a associação dos escores, o estudo aponta que o $\mathrm{p}$-valor é significativo para a correlação de antecedentes $x$ conhecimento, de forma que podemos sugerir que 0 paciente que apresenta um escore alto para antecedente e um baixo escore para o conhecimento tem uma predisposição para desenvolver as DRC, como pode ser verificado na tabela 5

Podemos observar que a maioria, $51.7 \%$, dos entrevistados estão classificadas em grau $V$ de PRÉ-DRC, já a segunda maior ocorrência está situada em grau VI com um percentual de $24.1 \%$, por sua vez $29.6 \%$ se encontram no grau IV e apenas $0.3 \%$ encontram-se no grau VIII, que foi o nível mais alto atingido e em relação ao menor grau atingido na pesquisa, o grau III, somam $0.7 \%$. Conforme o gráfico I.

Para uma melhor compreensão do grau de risco para DRC, foi classificada em risco leve, moderado e alto, no qual o risco leve corresponde $\leq$ ao grau III, já o risco moderado é > que grau III e < que grau VII, e por último o risco alto que vai do intervalo $\geq$ ao grau VII á $\leq$ grau X. Desta maneira, podemos aferir que cerca de $75 \%$ dos participantes encontram-se com risco moderado para DRC.

Esse estudo ratifica que não existe real diferença do PRÉ-DRC conforme Sexo, Idade, Estado civil, e Renda familiar, todos tem média entre 45 e 46 pontos.A diferença real ocorre no aspecto individual, uma vez que a correlação entre IMC e o PRÉ-DRC teve P-valor = 1757 (não significante), com coeficiente de correlação $r=0.0805$, já a correlação entre IDADE e o PRÉ-DRC teve $\mathrm{p}$-valor = 0.6730 (não significante), com coeficiente de correlação $r=0.025 \mathrm{I}$.

TABELA 5 - Correspondência entre os componentes do PRÉ-DRC em 286 idosos da UBS Condor. Belém/ PA, ano 2018.

\begin{tabular}{|c|c|c|c|}
\hline & $\begin{array}{l}\text { Antecedentes } \mathrm{x} \\
\text { Conhecimento }\end{array}$ & $\begin{array}{c}\text { Antecedentes x Estilo } \\
\text { de Vida }\end{array}$ & $\begin{array}{c}\text { Conhecimento } x \text { Estilo de } \\
\text { Vida }\end{array}$ \\
\hline Coef. e Correlação & 0.4755 & -0.0092 & 0.0182 \\
\hline Intervalo Confiança (IC95\%) & 0.17 a 0.38 & -0.12 a 0.11 & -0.10 a 0.13 \\
\hline Coeficiente R2 & 0.0759 & 0.0001 & 0.0003 \\
\hline p-valor & $<0.0001^{*}$ & 0.8756 & 0.7583 \\
\hline
\end{tabular}

Fonte: Elaborado pelos autores, Belém, 2018.

* Correlação Linear de Pearson. 


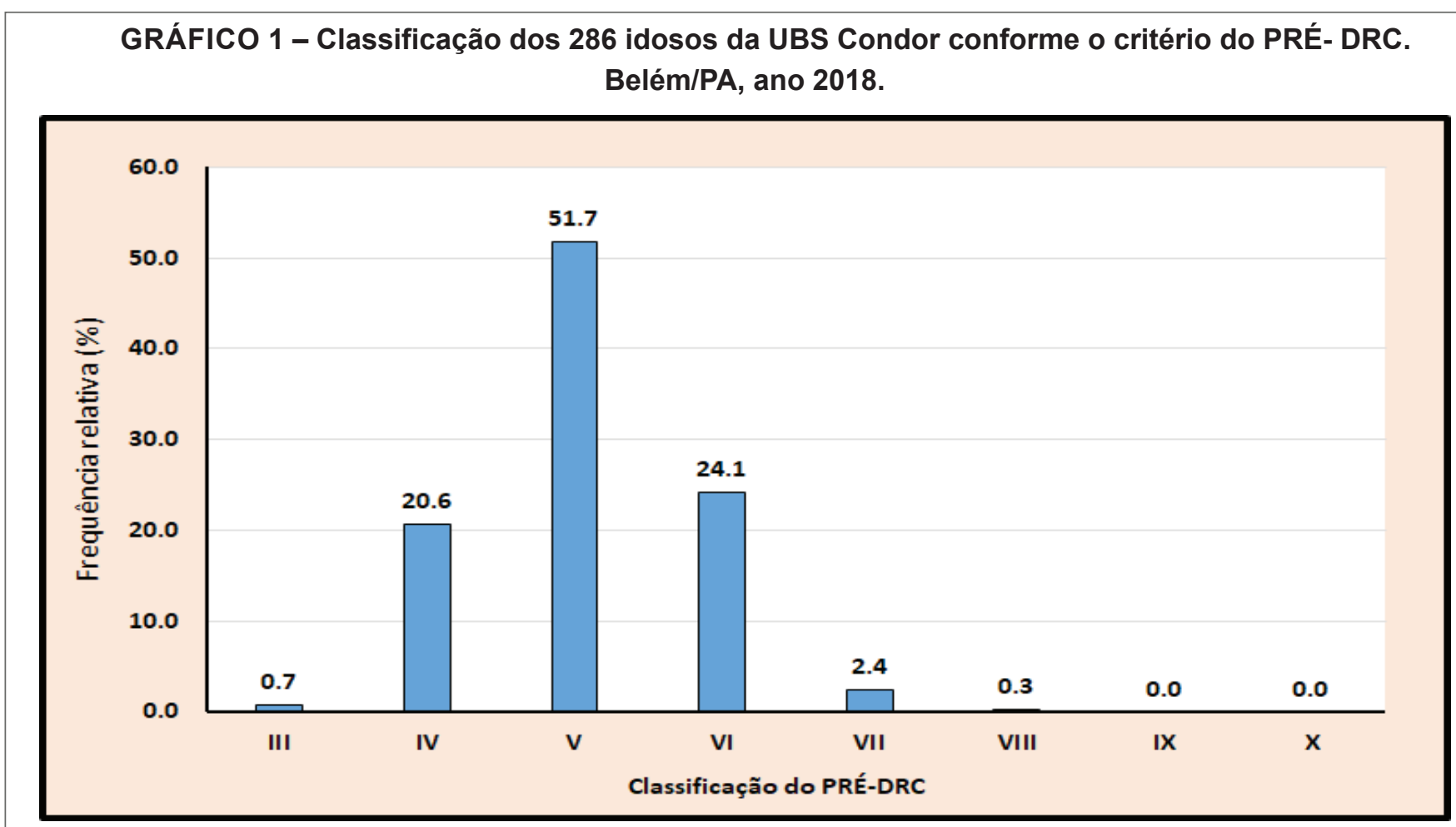

Fonte: Elaborado pelos autores, Belém, 2018.

\section{DISCUSSÃO}

A DRC atinge $10 \%$ da população mundial tendo uma estimativa de afetar um em cada cinco homens e uma em cada quatro mulheres com idade entre 65 e 74 anos, sendo que metade da população com 75 anos ou mais sofre algum grau da doença ${ }^{(8)}$. Esse estudo vem cooperar com dados encontrado na pesquisa, uma vez que a população estudada tem uma média de 65,5 anos, podendo caracterizar-se como uma população de risco para desenvolver DRC.

Outro dado que precisa ser levanto em consideração é a cor da pele, estudos apontam que a raça negra e parda tem uma maior chance para desenvolver HAS. Cerca de 95\% dos participantes do estudo podem desenvolver HAS se levar em consideração a cor da pele, no entanto sabese que não basta apenas a cor da pele para desenvolver tal doença, mas sim é a soma dos diversos fatores que interferem no processo saúde-doença para as DCNTs ${ }^{\left({ }^{9}\right.}$.

Duas das principais causas de DRC, o DM e a HAS, estão intimamente relacionadas ao sobrepeso ou obesidade, a obesidade é a causa de $65 \%$ a $75 \%$ das causas de HAS, que por sua vez é o principal fator de risco para a $D R C$, além do que a DM é um fator de risco pra desenvolver lesão renal. A maioria dos participantes apresentam sobrepeso, tendo paciente em obesidade de grau III. Logo é preciso fazer o rastreamento dos pacientes para fazer uma identificação precoce dos que apresentam risco para DRC (10,11).

Apesar de $44 \%$ afirmarem que fazem o controle da HAS, o conceito de controle sobre tal patologia para os participantes pode ter uma relação apenas medica- mentosa, uma vez quando analisamos os dados do IMC, demostra que a maioria dos participantes estão com sobrepeso, e sabe-se que o controle da HAS perpassa pelo tratamento medicamentoso e não medicamentoso, para que haja um controle dos fatores de risco para as complicações que podem levar o paciente a hospitalização.

Neste estudo $57 \%$ tem HAS e 34.8 tem DM e estão prédispostos a desenvolver algum problema renal, o que pode interferir na qualidade de vida desse paciente, pois precisará lidar com dois condicionantes crônicos, logo o mesmo necessita ter um maior esclarecimento sobre o controle da DCNT para que não ocorram complicações no seu quadro clinico, além de gerar um custo para o SUS ${ }^{(8)}$.

Outro fator que podemos ressaltar é que $52 \%$ dos participantes apresentam antecedentes familiares para HAS, fazendo necessária uma atenção relevante para esse fator na atenção primária, visto que pode ser realizado um rastreamento precoce para o grupo de risco, a fim prevenir os fatores modificáveis.

Torna-se preocupante que os usuários que apresentam risco para DRC não consigam identificar a causa da doença e nem a forma de prevenção. Essa situação pode ter uma relação direta com as orientações que são repassadas durante a consulta com a equipe multidisciplinar e às ações de saúde desenvolvidas, visto que, os usuários são cadastrados na UBS, porém apenas cerca de $10 \%$ refeririam que receberam algum tipo de informação sobre os fatores de risco para DRC.

A falta de informação a respeito das DRC dificulta a prevenção, o diagnóstico precoce e até mesmo o trata- 
mento ${ }^{(12)}$. Somado a esse desconhecimento dos fatores de risco para DRC, frisamos que quase $90 \%$ dos participantes afirmam que não receberam qualquer tipo de informação para prevenção por parte dos profissionais de saúde, contexto esse que é preocupante, visto que a Unidade de Saúde tem como premissa a promoção e prevenção à saúde, além de trabalhar com a rede de cuidado para as DCNT, desta forma os usuários deveriam ter recebido algum tipo de educação em saúde voltado para DRCs.

Quando solicitado para os participantes citarem os fatores que estão associados ao risco de DRC, o nosso estudo aponta que estatisticamente a automedicação, 0 cigarro e a bebida alcoólica foram significativos, demostrando assim o baixo conhecimento desses fatores por parte dos idosos.

Porém, sabe-se que $\circ$ tabagismo aumenta o risco renal tanto em indivíduos com DM e HAS quanto em não portadores dessas síndromes, sendo um dos principais fatores de risco modificáveis. $\mathrm{O}$ uso intenso de tabaco e a exposição cumulativa ao fumo é um fator de risco independente em homens e mulheres, sendo maléfico na progressão da DRC ${ }^{(13)}$. No que se refere ao uso de álcool, pode ser considerado fator de risco ou proteção de acordo com a quantidade. O uso diário de mais de duas doses de álcool aumenta o risco para DRC, enquanto o uso de menos de duas doses por dia atua como fator protetor $^{(14-15)}$.

Já a automedicação, que tem como conceito a forma de terapêutica na qual o paciente decide por si mesmo se deve fazer o tratamento medicamentoso e qual o produto que vai utilizar, essa prática pode acarretar em uma sobrecarga renal provocando agravos agudo e crônico(16). Outro fator que merece destaque são os produtos industrializados, pois tem sido um fator agravante para as DCNTs, e foi um dos fatores de estilo de vida significativo no estudo. $O$ consumo indiscriminado desses produtos pode tem uma relação com a renda familiar, uma vez que são produtos mais acessíveis, e os idosos do nosso estudo têm renda de dois salários mínimos, sendo que a principal fonte é a aposentadoria.

Nesse sentido, o produto industrializado tem sido um fator agravante para as DCNTs, e foi um dos fatores de estilo de vida significativo no estudo. $O$ consumo indiscriminado desses produtos pode tem uma relação com a renda familiar, uma vez que são produtos mais acessíveis, e os idosos do nosso estudo têm essa renda de dois salários mínimos, sendo que a principal fonte é aposentadoria.

Estudos sobre a condição financeira de idosos também relacionam o poder aquisitivo à qualidade de vida, por afetar 0 acesso a bens e serviços, por exemplo, alimentação de qualidade; essa alimentação tem uma satisfação social para idosos, indicando que o mesmo pode dá o sustendo para sua família, além de representar uma atitude de cumprimento da prescrição dos profissionais de saúde para compra e consumo de alimentos mais saudáveis ${ }^{(17)}$.

$\mathrm{O}$ alto consumo de carne vermelha, principalmente se for associada a gordura, tem sido apontado como um fator preocupante. As carnes, especialmente as vermeIhas e as processadas que possuem alta concentração de colesterol, ácidos graxos saturados e sódios, estão associados a incidências de doenças cardiovasculares, câncer e DRC ${ }^{(18)}$.

Além desses fatores de riscos já supracitados, a ausência de atividade física pode comprometer a qualidade de vida, aumentando a fragilidade dos idosos, além de aumentar os riscos para a obesidade, que acompanhada da má alimentação predispõe o individuo a desenvolver alguma DCNTs. Desta maneira é preciso que os profissionais da Atenção APS possam incluir no seu plano terapêutico o encorajamento dos idosos para praticar, pelo menos, três vezes na semana a caminhada, além de fazer o acompanhamento desses usuários, utilizando como ferramenta o IMC ${ }^{(19)}$.

Nosso estudo aponta que não há uma correlação da idade, sexo e IMC para a predisposição da DRC, mas sim que a relevância está na relação do grau de conhecimento, antecedente familiar e estilo de vida, que são fatores individuais, reforçando que o plano terapêutico precisa ser de acordo com a necessidade do usuário, levando em consideração os determinantes sócias em saúde, uma vez que exerce influência no estilo de vida e no nível de informação da população.

Ressaltamos que esse estudo é pioneiro na estratificação de risco para DRC, o qual levou em consideração três aspectos que foram: o histórico familiar e pessoal, 0 conhecimento e o estilo de vida. Fatores que necessitam ser abordados na avaliação dos idosos pela equipe multidisciplinar.

Percebe-se na literatura que o estudo de estratificação de risco é voltado para idosos com HAS, fazendo uma associação com as doenças cardiovasculares. O conhecimento do risco cardiovascular dos hipertensos auxilia no desenvolvimento e implementação de estratégias voltadas ao controle dos fatores de risco e prevenção de complicações ${ }^{(20)}$

Já quando se trata da estratificação de risco para as DRC, a maioria dos estudos trabalham na perspectiva de identificar o usuário que apresenta a DRC, como pode ser verificado em estudo que teve como objetivo avaliar o risco da DRC em amostra populacional de diabético, utilizando de marcadores biológicos para avaliação do exame de urina dos participantes, para determinar se o usuário apresentava ou não $D R C^{(21)}$.

Outra estratificação para DRC baseia-se na identi- 
ficação de grupos de risco, presença de alterações de sedimento urinário (microalbuminúria, proteinúria, hematúria e leucocitúria) e na redução da filtração glomerular avaliado pelo clearance de creatina. Essa é uma abordagem do Ministério da Saúde que de acordo com o clearance de creatinina, os indivíduos podem ser classificados em 6 estágios, que orientarão medidas preventivas e encaminhamento para especialista ${ }^{(22)}$.

Esses estudos de identificação precoce dos usuários com DRC são relevante para um tratamento precoce, no entanto o nosso estudo visa não apenas o tratamento, mas sim a prevenção e a mudança do estilo de vida dos usuários, uma vez quando se trabalha com a predisposição para DRC, pode fazer um planejamento de ações de acordo com a necessidade do usuário, possibilitando uma melhor qualidade de vida.

A estratificação dos indivíduos em subpopulações por riscos permite identificar indivíduos com necessidades de saúde semelhantes que devem ser atendidos com recursos e tecnologias específicos. Além disso, a estratificação de risco está associada à melhor qualidade da atenção à saúde e maior eficiência no uso dos recursos de saúde, já que diminui os erros associados à sub oferta de cuidados para portadores de maiores estratos de risco e a oferta em demasia para aqueles de menores estratos ${ }^{(23)}$.

Nesse sentido, o estudo pode nortear o rastreamen- to da população com predisposição em desenvolver a DRC, facilitando abordagem do plano terapêutico dos profissionais de saúde da Atenção Primaria à Saúde, possibilitando ações de prevenção, promoção e controle da saúde, a fim de evitar o agravamento do quadro clinico dos usuários de DCNTs, principalmente os de HAS e DM, de forma que os mesmo possa ter um aumento na perspectiva de vida com qualidade.

\section{CONCLUSÃO}

Estudos de identificação precoce dos usuários com DRC são relevante para um tratamento precoce, no entanto o nosso estudo visa não apenas o tratamento, mas sim a prevenção e a mudança do estilo de vida dos usuários, uma vez quando se trabalha com a predisposição para DRC, pode fazer um planejamento de ações de acordo com a necessidade do usuário, possibilitando uma melhor qualidade de vida.

Nesse sentido, o estudo pode nortear o rastreamento da população com predisposição em desenvolver a DRC, sendo uma ferramenta importante para estabelecer um plano terapêutico singular, condizente com a necessidade de cada usuário, a fim de evitar o agravamento do quadro clinico dos usuários de DCNTs, principalmente os de HAS e DM, de forma que os mesmo possa ter um aumento na perspectiva de vida com qualidade. 


\section{REFERÊNCIAS}

I.Kam Tao Li PI, Burdmann EA, Mehta RL. Acute Kidney Injury: a global alert. J. Bras. Nefrol. 2013; 35(I):I-5.DOI: I0.5935/0I0I2800.2013000I.

2. Soares FC,Aguiar IA, Carvalho NPF, Carvalho RF, Torres RA, Wellington S, Coelho FA, Oliveira MACA, Andrade FM, Costa JA. Prevalence of arterial hypertension and diabetes mellitus in carriers of chronic kidney disease in treatment conservator of the ubaense nefrologia service. Revista Cientifica Fagoc Saúde [Internet].2017[citado 2018 out. 26]; 2(2):2I-26. Disponível em: https://revista.fagoc.br/index.php/saude/article/view/232/243.

3.Bortolotto LA. Arterial hypertension and chronic renal failure. Rev. Bras Hipertens [Internet].2008[citado 2018 nov. 05];5(3):I52-I55. Disponível em: http://departamentos.cardiol.br/dha/revista/I5-3/09-hipertensao.pdf.

4. BRASIL. Ministério da Saúde. Secretaria de Atenção à Saúde. Departamento de Atenção Básica. Estratégias para o cuidado da pessoa com doença crônica: hipertensão arterial sistêmica[Internet]. Brasília (DF): Ministério da Saúde; 2014. (Cadernos de Atenção Básica; 37). [citado 2018 out. 2I]. Disponível em: http://bvsms.saude.gov.br/bvs/publicacoes/ estrategias_cuidado_pessoa_doenca_cronica_cab35.pdf.

5. Neto ACC. Biopsychosocial education development and quality of life among the elderly 65 and 75 years of age in Santa Ines city, Maranhão state, Brazil.Rev. COPEC. 2015. 19(22):283-287.DOI: 10.I4684/SHEWC.I5.20I5.283-287.

6. Souza MAH, Porto EF,Souza EL, Silva KI. Profile of lifestyle of older elderly persons. Rev. Bras. de Geriatria e Gerontologia 2016,19(5):819-826.DOI: 10.1590/180998232016019.150224.

7. Cochran WG. Sampling techniques. Willey e Sons. New York. 1986. 8. BRASIL. Portal Brasil. Doença renal crônica atinge $10 \%$ da população mundial. [Internet]. 2015.[citado 2018 out. 23]. Disponível em: http://www.brasil.gov.br/saude/2015/03/doenca-renal-cronica-atinge-I0-da-populacao-mundial.

9. Oliveira BLCA, Thomaz EBAF, Silva RA. The association between skin color/race and health indicators in elderly Brazilians: a study based on the Brazilian National Household Sample Survey (2008). Cad. Saúde Pública. 20I4; 30(7):I I5.DOI: |0.1590/0102-31।X0007|4|3.

I0.Miranda RNA,Carvalho EP, Amorim YR,Santos KS,Serrão FO. Getting to know the nutritional health of elderly people assisted by a non-governmental organization, Benevides / PA. Revista Conexão UEPG, Ponta Grossa. 2017; I3(3):5 I2529.DOI: 10.5212/Rev.Conexao.v.13.i3.0013.

II. Martins ALCL, Watanabe M, Fernandes SM, Fonseca CD, Vattimo MFF. Diabetes Mellitus: a risk factor for drug toxicity. Rev Esc Enferm USP. 2018;52:e03347. DOI: http://dx.doi. org/10.1590/S1980-220X2017033503347.

12. Menezes AF,Fontes ML,Correia MC,Santos DO,Andrade LCC, Santos AD. Impact of a new educational intervention on renal disease understanding in a high risk population. Revista ibero- am. Educ. investi. Enfermagem[Internet]. 2017[citado 2018 nov. 07];7(2): 13-20. Disponível: https://www.enfermeria21.com/revistas/aladefe/articulo/245/impacto-de-uma-intervencao-educativano-conhecimento-da-populacao-de-risco-sobre-a-doenca-renal/.

13. Orth SR, Hallan SI. Smoking:A Risk Factor for Progression of Chronic Kidney Disease and for Cardiovascular Morbidity and Mortality in Renal Patients-Absence of Evidence or Evidence of Absence? Clin J Am Soc Nephrol. 2008,3: 226-236. DOI: I0.22I5/CJN.03740907.

14. Perneger TV, Klag MJ,Whelton PK. Recreational drug use:A neglected risk factor for end-stage renal disease. Am J Kidney Dis. 200 I; 38(I):49-56. DOI: I0. I053/ajkd.200I.25 I8I.

15. Naghettini AV, Pereira ERS, Leão GT,Rocha MS,Batista SRR,Marques SM. Modifiable risk factors in chronic renal failure in the Family Health Strategy. Rev. Ciênc. Méd. [Internet]. 2017[citado 2018 nov. 27];25(3):99-I06.Disponível: https://seer.sis.puccampinas. edu.br/seer/index.php/cienciasmedicas/article/view/3।72/2485.

16. Schenkel EP, Mengue SS, Petrovick PR. Cuidados com os medicamentos. Rev. Florianópolis, $5^{\mathrm{a}}$ ed.: Ed. da UFSC; 2012.

17. FERREIRA MCG,Tura LFR, Silva RC, Ferreira MA Social representations of older adults regarding quality of life... Rev Bras Enferm. 2017; 70(4):806-I3.DOI: 10.I590/00347167-2017-0097.

18. Schneider BC, Duro SMS, Assunção MCF. Meat consumption by adults in southern Brazil: a population-based study. Ciênc. saúde coletiva.20I4; 19(8):3583-3592.DOI: I0.1590/|4|3-8|2320|4|98.|17020|3.

19. Dias EG, Souza BRS, Silva FES, Jesus M, Alves JCS. Estilo de vida de idosos usuários de uma unidade básica de saúde. Arq. Cienc. Saúde UNIPAR.20I7;20(2):I05-III.DOI: 10.25 I I0/arqsaude.v2Ii2.2017.5875.

20. Mendez RDR, Santos MA, Wysocki AD, Ribeiro BDAB, Stauffer LF, Duarte SJH. Cardiovascular risk stratification among hypertensive patients: the influence of risk factors. Rev Bras Enferm [Internet]. 2018;7I(4):I985-I99I.DOI: 10.1590/0034-7|67-2017-0528.

2I. Costa LRG,Novaes MR, Fernandes SEF, Luna LCG,Alexandre CS. avaliação do risco de doença renal crônica em uma amostra populacionalde diabéticos.Rev. Ciênc. Saúde Nova Esperança. 2014, I2(I):35-44.

22. Brasil. Ministério da Saúde. Secretaria de Atenção à Saúde. Departamento de Atenção Básica. Prevenção clínica de doenças cardiovasculares, cerebrovasculares e renais. [Internet]. 2006. [Acessado 2018 dez. 30]. Disponível em: http:// bvsms.saude.gov.br/bvs/publicacoes/abcad 14.pdf

23. Mendez RDR, Santos MA,Wysocki AD, Ribeiro BDAB, Stauffer LF, Duarte SJH. Cardiovascular risk stratification among hypertensive patients: the influence of risk factors. Rev Bras Enferm [Internet]. 20I8;7I(4):|985-9I. DOI: http://dx.doi. org/10.1590/0034-7/67-2017-0528.

Recebido: $2020-07-30$ Aceito: 2020-09-14 